\title{
Эффективная масса плотности состояний и подвижность носителей заряда в гетероэпитаксиальных пленках теллурида висмута и твердых растворах $\mathrm{Bi}_{0.5} \mathrm{Sb}_{1.5} \mathrm{Te}_{3}$
}

\author{
(С) Л.Н. Лукьянова, Ю.А. Бойков, О.А. Усов, В.А. Данилов \\ Физико-технический институт им. А.Ф. Иоффе Российской академии наук, \\ 194021 Санкт-Петербург, Россия \\ E-mail: lidia.lukyanova@mail.ioffe.ru
}

(Получена 12 декабря 2016 г. Принята к печати 19 декабря 2016 г.)

\begin{abstract}
Показано, что коэффициент термоэдс $\alpha$, параметр мощности $\alpha^{2} \sigma$ и эффективная масса плотности состояний $m / m_{0}$ в гетероэпитаксиальных пленках твердых растворов $\mathrm{Bi}_{0.5} \mathrm{Sb}_{1.5} \mathrm{Te}_{3}$ возрастают по сравнению с объемными термоэлектриками. Рост и слабые температурные зависимости этих величин приводят к увеличению параметра материала, пропорционального эффективной массе, подвижности и термоэлектрической эффективности. Характер изменения $\alpha, \alpha^{2} \sigma$ и $\mathrm{m} / \mathrm{m}_{0}$ определяется особенностями механизма рассеяния носителей заряда, анизотропией поверхности постоянной энергии и возможным влиянием топологических поверхностных состояний дираковских фермионов в пленках.
\end{abstract}

DOI: $10.21883 /$ FTP.2017.06.44543.02

\section{1. Введение}

Наноструктурированые пленки на основе халькогенидов висмута и сурьмы характеризуются высокой термоэлектрической эффективностью $[1,2]$ и являются перспективными термоэлектриками для применения в устройствах нового поколения. Повышение термоэлектрической эффективности в пленках халькогенидов обеспечивается за счет селективного рассеяния фононов на межфазных и межкристаллитных границах, что приводит к снижению теплопроводности при высоком параметре мощности. Анализ температурных зависимостей коэффициента термоэдс и удельной электропроводности гетероэпитаксиальных пленок $\mathrm{Bi}_{2} \mathrm{Te}_{3}, \mathrm{Bi}_{0.5} \mathrm{Sb}_{1.5} \mathrm{Te}_{3}$ субмикронной толщины и соответствующих объемных термоэлектриков в интервале температур 80-300 К позволяет установить зависимости между термоэлектрическими свойствами и величинами эффективной массы плотности состояний $m / m_{0}$ и подвижности $\mu_{0}$ в параболической модели энергетического спектра с учетом изменения механизма рассеяния носителей заряда.

\section{2. Термоэлектрические свойства}

На рис. 1 приведены зависимости коэффициента термоэдс $\alpha$ и электропроводности $\sigma$ в интервале температур 80-300 K для пленок $\mathrm{Bi}_{2} \mathrm{Te}_{3}, \mathrm{Bi}_{0.5} \mathrm{Sb}_{1.5} \mathrm{Te}_{3}$ и объемного твердого раствора того же состава.

Гетероэпитаксиальные пленки были выращены методом горячей стенки, объемные образцы - методом направленной кристаллизации с прецизионной регулировкой температуры. Сравнение температурных зависимостей коэффициента термоэдс $\alpha$ в пленках твердых растворов $\mathrm{Bi}_{0.5} \mathrm{Sb}_{1.5} \mathrm{Te}_{3}$ с объемными образцами с близкими значениями $\alpha$ при комнатной температуре показывает, что в пленках коэффициент термоэдс выше в низкотемпературной области, т.е. наблюдается небольшое ослабление зависимости $\alpha(T)$ с уменьшением температуры (рис. 1, кривые $1-3$ ), что приводит к уменьшению угловых коэффициентов $d \ln \alpha / d \ln T$ от 1 в объемных твердых растворах до 0.65 и 0.4 в пленках (кривые $5,1,2$ ). В пленке $\mathrm{Bi}_{2} \mathrm{Te}_{3}$ наблюдалось резкое снижение $\alpha$ с температурой (рис. 1, кривая 4). Электропроводность $\sigma$ возрастает сильнее при снижении температуры в объемных твердых растворах, чем в пленках (рис. 1, кривые 5,7), однако с повышением $\alpha$ до 233 и $242 \mathrm{м \kappa В} \cdot \mathrm{K}^{-1}$ величины электропроводности в пленке и объемном образце становятся близкими, что объясняется слабым рассеянием носителей заряда на

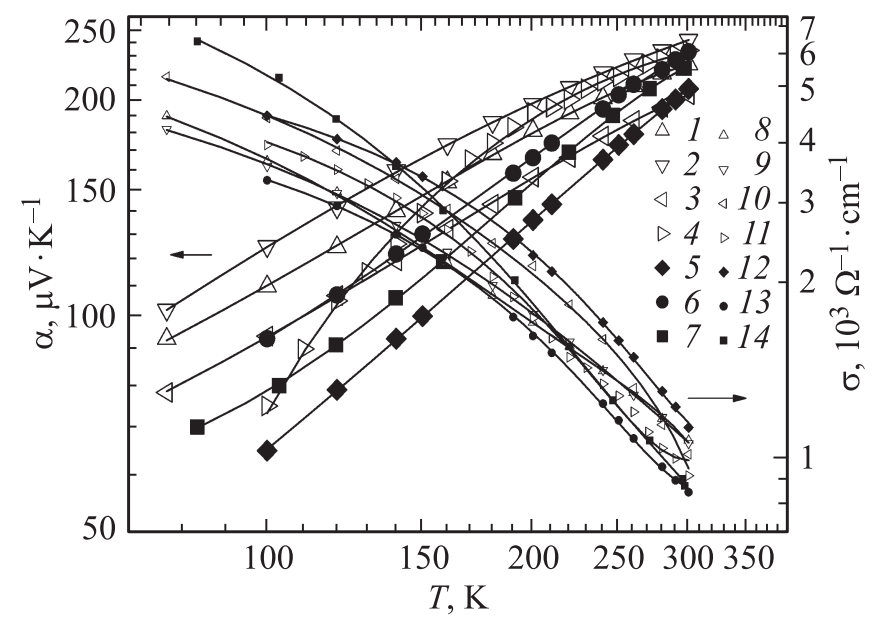

Рис. 1. Температурные зависимости коэффициента термоэдс $\alpha(1-7)$ и электропроводности $\sigma \quad(8-14)$ от температуры в эпитаксиальных пленках $(1-4,8-11)$ и объемных образцах $(5-7,12-14)$ твердого раствора $\mathrm{Bi}_{0.5} \mathrm{Sb}_{1.5} \mathrm{Te}_{3} \quad(1-3,5-7,8-10,12-14) \quad$ и теллурида висмута $\mathrm{Bi}_{2} \mathrm{Te}_{3}(4,11) . \alpha, \mu \mathrm{VK}^{-1}: 1-224,2-242,3-203$, $4-234,5-207,6-233,7-221$. 


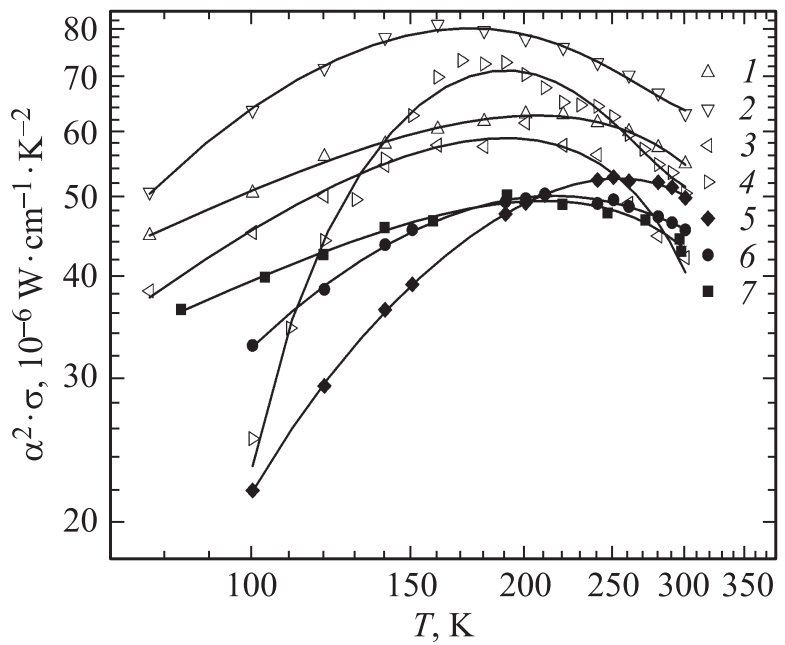

Рис. 2. Температурные зависимости параметра мощности от температуры в пленках (1-4) и объемных образцах (5-7) твердого раствора $\mathrm{Bi}_{0.5} \mathrm{Sb}_{1.5} \mathrm{Te}_{3}(1-3,5-7)$ и теллурида висмута $\mathrm{Bi}_{2} \mathrm{Te}_{3}(4)$.

межфазных и межкристаллитных границах в пленках. Отсюда следует, что увеличение параметра мощности в пленках $\mathrm{Bi}_{0.5} \mathrm{Sb}_{1.5} \mathrm{Te}_{3}$ при слабой зависимости $\alpha^{2} \sigma$ от $T$ в низкотемпературной области определяется в основном ростом величины и ослаблением температурной зависимости термоэдс (рис. 1,2).

\section{3. Эффективная масса и подвижность}

Результаты исследования термоэлектрических и гальваномагнитных свойств в эпитаксиальных пленках и объемных термоэлектриках позволяют определить усредненную эффективную массу плотности состояний $m / m_{0}$ и подвижность носителей заряда $\mu_{0}$ с учетом эффективного параметра рассеяния $r_{\text {eff }}[3,4]$. Расчеты показали, что в пленках $r_{\text {eff }}$ сильнее отличается от значения -0.5 , характерного для акустического механизма рассеяния, чем в объемных термоэлектриках, и изменяется от -0.6 до -0.9 . Полученные величины $r_{\text {eff }}$ указывают на изменение энергетической зависимости времени релаксации в пленках по сравнению с объемными термоэлектриками для модели, в которой $\tau$ имеет степенную зависимость от энергии в виде $\tau=\tau_{0} E^{r}$, где $\tau_{0}$ не зависит от энергии $E$.

Эффективная масса $m / m_{0}$ в пленках твердого раствора $\mathrm{Bi}_{0.5} \mathrm{Sb}_{1.5} \mathrm{Te}_{3}$ выше, чем в объемных образцах, и имеет слабую зависимость от температуры в отличие от пленки $\mathrm{Bi}_{2} \mathrm{Te}_{3}$, в которой $m / m_{0}$ резко уменьшается при низких температурах, как и термоэдс (рис. 3, кривые $1-4$, рис. 1, кривая 4). Увеличение $m / m_{0}$ в объемном образце, для которого $\alpha=203$ мкВ $\cdot \mathrm{K}^{-1}$ при комнатной температуре (рис. 3 , кривая 5), связано с повышением концентрации носителей по сравнению с другими образцами.
Зависимости $m / m_{0}$ от $T$ в области низких температур слабее, как и зависимости $\alpha$ и $\alpha^{2} \sigma$ от температуры, по сравнению с объемными образцами (рис. 1-3) в основном вследствие особенностей механизма рассеяния носителей заряда [3] и изменения параметров поверхности постоянной энергии в пленках [5]. Кроме того, на характер рассматриваемых температурных зависимостей могут влиять топологические поверхностные состояния дираковских фермионов в халькогенидных пленках, что согласуется с результатами барических исследований в слоях $\mathrm{Bi}_{2} \mathrm{Te}_{3}$ и подтверждается исследованиями рамановского рассеяния под давлением [6].

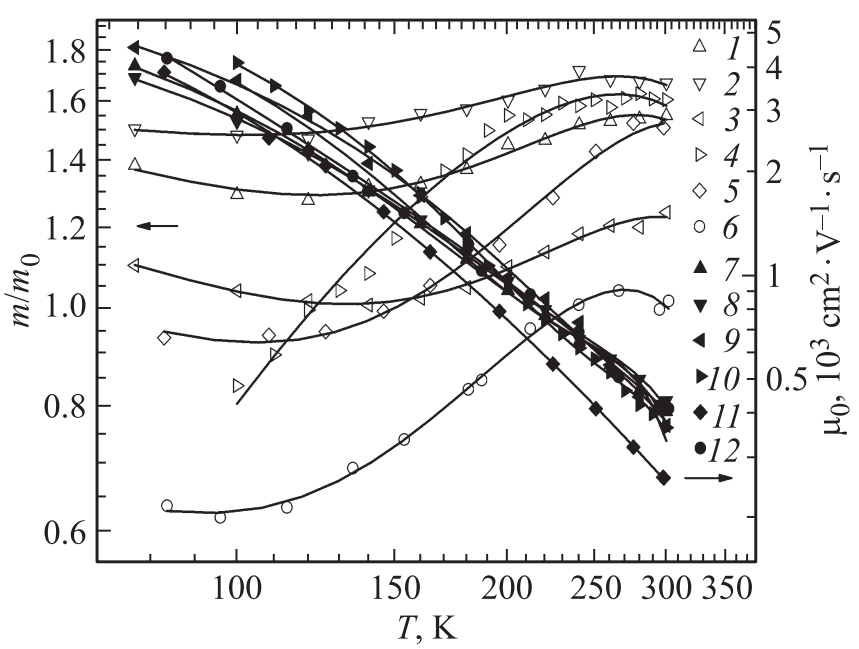

Pис. 3. Температурные зависимости эффективной массы плотности состояний $m / m_{0}(1-6)$ и подвижности носителей заряда $\mu_{0}(7-12)$ в эпитаксиальных пленках $(1-4,7-10)$ и объемных образцах $(5,6,11,12)$ твердого раствора $\mathrm{Bi}_{0.5} \mathrm{Sb}_{1.5} \mathrm{Te}_{3}(1-3,5-9,11,12)$ и теллурида висмута $\mathrm{Bi}_{2} \mathrm{Te}_{3}(4,10)$.

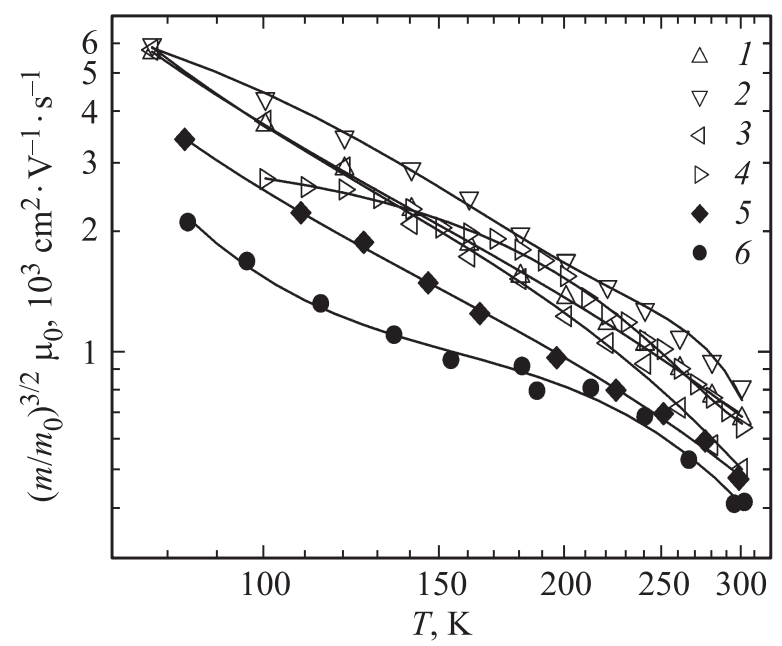

Рис. 4. Температурные зависимости параметра $\left(m / m_{0}\right)^{3 / 2} \mu_{0}$ в эпитаксиальных пленках (1-4) и объемных образцах $(5,6)$ твердого раствора $\mathrm{Bi}_{0.5} \mathrm{Sb}_{1.5} \mathrm{Te}_{3}(1-3,6,7)$ и теллурида висмута $\mathrm{Bi}_{2} \mathrm{Te}_{3}(4,5)$. 


\section{4. Параметр материала $\left(m / m_{0}\right)^{3 / 2} \mu_{0}$}

Параметр материала в виде $\left(m / m_{0}\right)^{3 / 2} \mu_{0}$, пропорциональный термоэлектрической эффективности $Z$, выше в пленках, чем в объемных термоэлектриках (рис. 4), и определяется ростом эффективной массы $\mathrm{m} / \mathrm{m}_{0}$ и ее слабой температурной зависимостью (рис. 3, кривые 1,2$)$. По оценкам величина термоэлектрической эффективности $Z$ в гетероэпитаксиальных пленках твердого раствора $\mathrm{Bi}_{0.5} \mathrm{Sb}_{1.5} \mathrm{Te}_{3}$ составляет $3.85 \cdot 10^{-3} \mathrm{~K}^{-1}$ при температурах 180-200 К для этих образцов. Полученная величина $Z$ в гетероэпитаксиальных пленках на $60-70 \%$ выше, чем в стандартных объемных материалах, и выше на 20\% по сравнению с многокомпонентными объемными термоэлектриками, оптимизированными для температур ниже $200 \mathrm{~K}$ [7].

\section{5. Заключение}

Исследования термоэлектрических свойств в гетероэпитаксиальных пленках $\mathrm{Bi}_{2} \mathrm{Te}_{3}$ и $\mathrm{Bi}_{0.5} \mathrm{Sb}_{1.5} \mathrm{Te}_{3}$ показали, что в твердом растворе $\mathrm{Bi}_{0.5} \mathrm{Sb}_{1.5} \mathrm{Te}_{3}$ коэффициент термоэдс, параметр мощности и эффективная масса плотности состояний возрастают, а подвижность слабо снижается по сравнению с объемными термоэлектриками, что приводит к росту параметра материала $\left(m / m_{0}\right)^{3 / 2} \mu_{0}$, пропорционального термоэлектрической эффективности $Z$. Обнаруженные особенности поведения температурных зависимостей указанных величин определяются изменением механизма рассеяния носителей заряда и анизотропией поверхности постоянной энергии $[3,6]$. Также возможно влияние топологических поверхностных состояний дираковских фермионов в пленках халькогенидов, что согласуется с результатами барических исследований в тонких слоях $\mathrm{Bi}_{2} \mathrm{Te}_{3}$.

Исследования выполнены за счет гранта Российского научного фонда (проект № 16-42-01067).

\section{Список литературы}

[1] L. Hicks, M. Dresselhaus. Phys. Rev. B, 47, 16631 (1993).

[2] B. Poudel, Q. Hao, Y. Ma, Y. Lan, A. Minnich, B. Yu, X. Yan, D. Wang, A. Muto, D. Vashaee, X. Chen, J. Liu, M.S. Dresselhaus, G. Chen, Z. Ren. Science, 320, 634 (2008).

[3] L.N. Lukyanova, Yu.A. Boikov, V.A. Danilov, O.A. Usov, M.P. Volkov, V.A. Kutasov. Semicond. Sci. Technol., 30, 015011 (2015).

[4] Л.Н. Лукьянова, В.А. Кутасов, В.В. Попов, П.П. Константинов. ФТТ, 46, 1366 (2004).

[5] L.N. Lukyanova, Yu.A. Boikov, V.A. Danilov, M.P. Volkov, V.A. Kutasov. J. Electron. Mater., 42, 1796 (2013).

[6] S.V. Ovsyannikov, N.V. Morozova, I.V. Korobeinikov, L.N. Lukyanova, A.Y. Manakov, A.Y. Likhacheva, A.I. Ancharov, A.P. Vokhmyanin, I.F. Berger, O.A. Usov, V.A. Kutasov, V.A. Kulbachinskii, T. Okada, V.V. Shchennikov. Appl. Phys. Lett., 106, 143901 (2015).

[7] Л.Н. Лукьянова, В.А. Кутасов, П.П. Константинов, В.В. Попов. ФТТ, 52, 1492 (2010).

Редактор Г.А. Оганесян

\section{The Density of States Effective Mass and Carrier Mobility in Heteroepitaxial Films of Bismuth Telluride and $\mathbf{B i}_{0.5} \mathbf{S b}_{1.5} \mathbf{T e}_{3}$ Solid Solution}

\author{
L.N. Lukyanova, A.Yu. Boikov, O.A. Usov, V.A. Danilov \\ loffe Institute, \\ 194021 St. Petersburg, Russia
}

\begin{abstract}
It is shown that the Seebeck coefficient $\alpha$, the power factor $\alpha^{2} \sigma$ and the density of states effective mass $m / m_{0}$ in heteroepitaxial films of $\mathrm{Bi}_{0.5} \mathrm{Sb}_{1.5} \mathrm{Te}_{3}$ solid solution are increased compared to the bulk thermoelectrics. Increasing and weak temperature dependences of these parameters lead to the rise of the material parameter, which is proportional to the effective mass, the mobility of charge carriers and the figure of merit $Z$.

The changes of the $\alpha, \alpha^{2} \sigma$ and $m / m_{0}$ values are determined by the peculiarities of charge carrier scattering mechanism, the anisotropy of the constant energy surface and the possible influence of topological surface states of Dirac fermions in the films.
\end{abstract}

